\title{
On the Efficiency of Information-Assisted Search for Parking Space: A Game-Theoretic Approach*
}

\author{
Evangelia Kokolaki, Merkourios Karaliopoulos, and Ioannis Stavrakakis \\ Department of Informatics and Telecommunications, \\ National \& Kapodistrian University of Athens \\ Ilissia, 15784 Athens, Greece \\ \{evako, mkaralio, ioannis\} @di.uoa.gr
}

\begin{abstract}
This paper seeks to systematically explore the efficiency of the uncoordinated information-assisted parking search in urban environments with two types of parking resource facilities: inexpensive but limited facilities (public) and expensive yet unlimited ones (private); an additional cruising cost is incurred when deciding for a public facility but failing to actually utilize one. Drivers decide whether to go for the public or directly for the private facilities, assuming perfect knowledge of prices and costs, total parking capacities and demand; the latter information can be broadcast by an ideal centralized information dissemination mechanism, assisting the otherwise uncoordinated parking search process. Drivers are viewed as strategic decision-makers that aim at minimizing the cost of the acquired parking spot. We formulate the resulting game as an instance of resource selection games and derive its Nash equilibria and their dependence on the environmental parameters such as the parking demand and supply as well as the pricing policy. The cost at the equilibrium states is compared to that under the optimal resource assignment (dictated to the drivers directly by an ideal centralized scheme) and conditions are derived for minimizing the related price of anarchy. Finally, the numerical results and the presented discussion provide hints for the practical management and pricing of public and private parking resources.
\end{abstract}

\section{Introduction}

The tremendous increase of urbanization necessitates the efficient and environmentally sustainable management of urban processes and operations. The (pilot) implementation of solutions and pioneering ideas from the area of information and communication technologies presents a response to this need paving the way for the so-called "smart cities". The search for available parking space is among the daily routine processes that can benefit from this new kind of city environments. In particular, transportation engineers have developed parking assistance systems, realized through information dissemination mechanisms to alleviate not only the traffic congestion problems that stem from the blind parking search but also the resulting environmental burden. Common to

\footnotetext{
* This work has been supported in part by the European Commission IST-FET project RECOGNITION (FP7-IST-257756), the European Commission under the Network of Excellence in Internet Science project EINS (FP7-ICT-288021) and the University of Athens Special Account of Research Grants no 10812.
} 
these systems is the exploitation of wireless communications and information sensing technologies to collect and broadcast (in centralized mechanisms, i.e., in [18], [20]) or share (in distributed systems, i.e., in [8], [10]) information about the availability of parking space and the demand for it within the search area. This information is then used to steer the parking choices of drivers in order to reduce the effective competition over the parking space and make the overall search process efficient. Additionally, the implementation of smart demand-responsive pricing policies on the parking facilities intends to improve parking availability in overused parking zones and reduce double-parking and cruising phenomena (i.e., in [1]).

This paper seeks to systematically explore the fundamental limits on the efficiency of these parking assistance systems. To this end, it ideally assumes that drivers become completely aware of the competition intensity, parking capacity and applied pricing policies on the parking facilities. Thus, the particular questions that become relevant for this exploration are: How do these three parameters modulate drivers' parking choices? How do they affect the cost that drivers incur and the revenue accruing for the parking service operator?

We formulate the parking spot selection problem as an instance of resource selection games in Section 2. We view the drivers as rational selfish agents that try to minimize the cost they pay for acquired parking space. The drivers choose to either compete for the cheaper but scarce on-street parking spots or head for the more expensive private parking $\operatorname{lot}(\mathrm{s}) \sqrt[1]{1}$. In the first case, they run the risk of failing to get a spot and having to $a$ posteriori take the more expensive alternative, this time suffering the additional cruising cost in terms of time, fuel consumption (and stress) of the failured attempt. Drivers make their decisions drawing on perfect information about the number of drivers, the availability of parking spots and the pricing policy, which is broadcast from the parking service operator. In Section 3, we derive the equilibrium behaviors of the drivers and compare the induced social cost against the optimal one via the Price of Anarchy metric. Most importantly, in Section 4, we show that the optimization of the equilibrium social cost is feasible by properly choosing the pricing and location of the private parking facilities. We outline related research in Section 5 and we close the discussion in Section 6 iterating on the model assumptions.

\section{The Parking Spot Selection Game}

In the parking spot selection game, the set of players consists of drivers who circulate within the center area of a big city in search of parking space. Typically, in these regions, parking is completely forbidden or constrained in whole areas of road blocks so that the real effective curbside is significantly limited (see Fig. 1). The drivers have to decide whether to drive towards the scarce low-cost (controlled) public parking spots or the over-dimensioned and more expensive private parking lot (we see all local lots collectively as one). All parking spots that lie in the same public or private area are assumed to be of the same value for the players. Especially the on-street parking spots

\footnotetext{
${ }^{1}$ Hereafter in the paper, the terms public parking spots and private parking facilities denote onstreet parking spots and parking lots, respectively. Their context in this paper should not be confused with that of public/private goods in economics.
} 


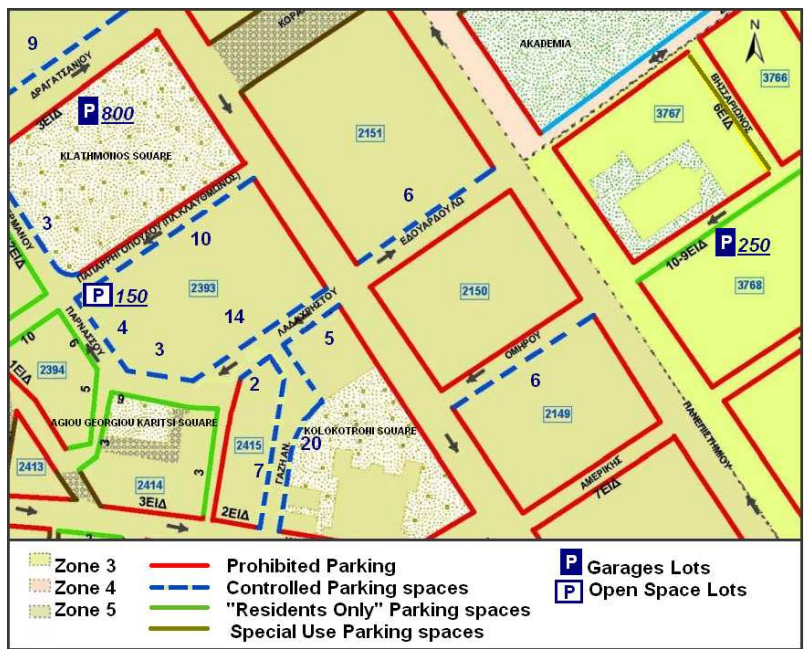

Fig. 1. Parking map of the centre area of Athens, Greece. Dashed lines show metered controlled (public) parking spots, whereas "P" denotes private parking facilities. The map illustrates, as well, the capacity of both parking options.

are considered quite close to eachother, resulting in practically similar driving times to them and walking times from them to the drivers' ultimate destinations. Thus, the decisions are made on the two sets of parking spots rather than individual set items.

We observe drivers' behavior within a particular time window over which they reach this parking area. In general, these synchronization phenomena in drivers' flow occur at specific time zones during the day [2]. Herein, we account for morning hours or driving in the area for business purposes coupled with long parking duration. Thus, the collective decision making on parking space selection can be formulated as an instance of the strategic resource selection games, whereby $N$ players (i.e., drivers) compete against each other for a finite number of common resources (i.e., public parking spots) [6]. More formally, the one-shol] parking spot selection game is defined as follows:

Definition 1. A Parking Spot Selection Game is a tuple $\Gamma=\left(\mathcal{N}, \mathcal{R},\left(A_{i}\right)_{i \in \mathcal{N}}\right.$, $\left(w_{j}\right)_{j \in(\text { pub,priv })) \text {, where: }}$

$-\mathcal{N}=\{1, \ldots, N\}, N>1$ is the set of drivers who seek for parking space,

$-\mathcal{R}=\mathcal{R}_{\text {pub }} \cup \mathcal{R}_{\text {priv }}$ is the set of parking spots; $\mathcal{R}_{\text {pub }}$ is the set of public spots, with $R=\left|\mathcal{R}_{\text {pub }}\right| \geq 1 ; \mathcal{R}_{\text {priv }}$ the set of private spots, with $\left|\mathcal{R}_{\text {priv }}\right| \geq N$,

- $A_{i}=\{$ public, private $\}$, is the action set for each driver $i \in \mathcal{N}$,

- $w_{\text {pub }}(\cdot)$ and $w_{\text {priv }}(\cdot)$ are the cost functions of the two actions, respectively.

The parking spot selection game comes under the broader family of congestion games. The players' payoffs (here: costs) are non-decreasing functions of the number of players competing for the parking capacity rather than their identities and common

\footnotetext{
${ }^{2}$ The study of the dynamic variant of the game is a natural direction for future work.
} 
to all players. More specifically, drivers who decide to compete for the public parking space undergo the risk of not being among the $R$ winner-drivers to get a spot. In this case, they have to eventually resort to private parking space, only after wasting extra time and fuel (plus patience supply) on the failed attempt. The expected cost of the action public, $w_{\text {pub }}: A_{1} \times \ldots \times A_{N} \rightarrow \mathbb{R}$, is therefore a function of the number of drivers $k$ taking it, and is given by

$$
w_{p u b}(k)=\min (1, R / k) c_{p u b, s}+(1-\min (1, R / k)) c_{p u b, f}
$$

where $c_{p u b, s}$ is the cost of successfully competing for public parking space, whereas $c_{p u b, f}=\gamma \cdot c_{p u b, s}, \gamma>1$, is the cost of competing, failing, and eventually paying for private parking space.

On the other hand, the cost of private parking space is fixed

$$
w_{\text {priv }}(k)=c_{\text {priv }}=\beta \cdot c_{\text {pub }, s}
$$

where $1<\beta<\gamma$, so that the excess $\cos t \delta \cdot c_{p u b, s}$, with $\delta=\gamma-\beta>0$, reflects the actual cost of cruising and the "virtual" cost of wasted time till eventually heading to the private parking space.

We denote an action profile by the vector $a=\left(a_{i}, a_{-i}\right) \in \times{ }_{k=1}^{N} A_{k}$, where $a_{-i}$ denotes the actions of all other drivers but player $i$ in the profile $a$. Besides the two pure strategies reflecting the pursuit of public and private parking space, the drivers may also randomize over them. In particular, if $\Delta\left(A_{i}\right)$ is the set of probability distributions over the action set of player $i$, a player's mixed action corresponds to a vector $p=$ $\left(p_{\text {pub }}, p_{\text {priv }}\right) \in \Delta\left(A_{i}\right)$, where $p_{\text {pub }}$ and $p_{\text {priv }}$ are the probabilities of the pure actions, with $p_{p u b}+p_{\text {priv }}=1$, while its cost is a weighted sum of the cost functions $w_{p u b}(\cdot)$ and $w_{\text {priv }}(\cdot)$ of the pure actions.

In the following section, we derive the game-theoretic analysis of the particular game formulation looking into both the stable and optimal operational conditions as well as the respective costs incurred by the players.

\section{Game Analysis}

Ideally, the players determine their strategy under complete knowledge of those parameters that shape their cost. Given the symmetry of the game, the additional piece of information that is considered available to the players, besides the number of vacant parking spots and the employed pricing policy, is the level of parking demand, i.e., the number of drivers searching for parking space. We draw on concepts from [15] and theoretical results from [6, 9] to derive the equilibrium strategies for the game $\Gamma$ and assess their (in)efficiency.

\subsection{Pure Equilibrium Strategies}

Existence: The parking spot selection game constitutes a symmetric game, where the action set is common to all players and consists of two possible actions, public and private. Cheng et al. have shown in ([9], Theorem 1) that every symmetric game with two strategies has an equilibrium in pure strategies. 
Derivation: Due to the game's symmetry, the full set of $2^{N}$ different action profiles maps into $N+1$ different action meta-profiles. Each meta-profile $a(m), m \in[0, N]$ encompasses all $\left(\begin{array}{l}N \\ m\end{array}\right)$ different action profiles that result in the same number of drivers competing for on-street parking space. The expected costs for these $m$ drivers and for the $N-m$ ones choosing directly the private parking lot alternative are functions of $a(m)$ rather than the exact action profile.

In general, the cost $c_{i}^{N}\left(a_{i}, a_{-i}\right)$ for driver $i$ under the action profile $a=\left(a_{i}, a_{-i}\right)$ is

$$
c_{i}^{N}\left(a_{i}, a_{-i}\right)=\left\{\begin{array}{l}
w_{\text {pub }}\left(N_{\text {pub }}(a)\right), \text { for } a_{i}=\text { public } \\
w_{\text {priv }}\left(N-N_{\text {pub }}(a)\right), \text { for } a_{i}=\text { private }
\end{array}\right.
$$

where $N_{p u b}(a)$ is the number of competing drivers for on-street parking under action profile $a$. Equilibrium action profiles combine the players' best-responses to their opponents' actions. Formally, an action profile $a=\left(a_{i}, a_{-i}\right)$ is a pure Nash equilibrium if for all $i \in \mathcal{N}$ :

$$
a_{i} \in \arg \min _{a_{i}^{\prime} \in A_{i}}\left(c_{i}^{N}\left(a_{i}^{\prime}, a_{-i}\right)\right)
$$

so that no player has anything to gain by changing her decision unilaterally.

Therefore, to derive the equilibrium states, we determine the conditions on $N_{p u b}$ that break the equilibrium definition and reverse them. More specifically, given an action profile $a$ with $N_{p u b}(a)$ competing drivers, a player gains by changing her decision to play action $a_{i}$ in two circumstances:

$$
\begin{gathered}
\text { when } a_{i}=\text { private and } w_{\text {pub }}\left(N_{\text {pub }}(a)+1\right)<c_{\text {priv }} \\
\text { when } a_{i}=\text { public and } w_{\text {pub }}\left(N_{\text {pub }}(a)\right)>c_{\text {priv }}
\end{gathered}
$$

Taking into account the relation between the number of drivers and the available on-street parking spots, $R$, we can postulate the following Lemma:

Lemma 1. In the parking spot selection game $\Gamma$, a driver is motivated to change her action $a_{i}$ in the following circumstances:

$$
\begin{aligned}
& \text { - } a_{i}=\text { private and } N_{\text {pub }}(a)<R \leq N \text { or } \\
& R \leq N_{\text {pub }}(a)<N_{0}-1 \leq N \text { or } \\
& N_{p u b}(a)<N \leq R \\
& \text { - } a_{i}=\text { public and } R<N_{0}<N_{\text {pub }}(a) \leq N
\end{aligned}
$$

where $N_{0}=\frac{R(\gamma-1)}{\delta} \in \mathbb{R}$.

Proof. Conditions (7) and (9) are trivial. Since the current number of competing vehicles is less than the on-street parking capacity, every driver having originally chosen the private parking option has the incentive to change her decision due to the price differential between $c_{p u b, s}$ and $c_{p r i v}$.

When $N_{p u b}(a)$ exceeds the public parking supply, a driver who has decided to avoid competition, profits from switching her action when (5) holds, which combined with (1), yields (8). Similarly, a driver that first decides to compete, profits by switching her action if (6) holds, which combined with (1), yields (10). 
The following Theorem for the pure Nash equilibria of the parking spot selection game may now be stated.

Theorem 1. A parking spot selection game has:

(a) one Nash equilibrium a* with $N_{p u b}\left(a^{*}\right)=N_{p u b}^{N E, 1}=N$, if $N \leq N_{0}$ and $N_{0} \in \mathbb{R}$

(b) $\left(\begin{array}{c}N \\ \left\lfloor N_{0}\right\rfloor\end{array}\right)$ Nash equilibrium profiles $a^{\prime}$ with $N_{p u b}\left(a^{\prime}\right)=N_{p u b}^{N E, 2}=\left\lfloor N_{0}\right\rfloor$, if $N>N_{0}$ and $N_{0} \in(R, N) \backslash \mathbb{N}^{*}$

(c) $\left(\begin{array}{c}N \\ N_{0}\end{array}\right)$ Nash equilibrium profiles $a^{\prime}$ with $N_{p u b}\left(a^{\prime}\right)=N_{p u b}^{N E, 2}=N_{0}$ and $\left(\begin{array}{c}N \\ N_{0}-1\end{array}\right)$ Nash equilibrium profiles $a^{\star}$ with $N_{p u b}\left(a^{\star}\right)=N_{p u b}^{N E, 3}=N_{0}-1$, if $N>N_{0}$ and $N_{0} \in[R+1, N] \cap \mathbb{N}^{*}$.

Proof. Theorem 1 follows directly from (4) and Lemma11 The game has two equilibrium conditions on $N_{p u b}$ for $N>N_{0}$ with integer $N_{0}$ (case c), or a unique equilibrium condition, otherwise (cases a, b).

Efficiency: The efficiency of the equilibrium action profiles resulting from the strategically selfish decisions of the drivers is assessed through the broadly used metric of the Price of Anarchy [15]. It expresses the ratio of the social cost in the worst-case equilibria over the optimal social cost under ideal coordination of the drivers' strategies.

Proposition 1. In the parking spot selection game, the pure Price of Anarchy equals:

$$
\mathrm{P} \circ \mathrm{A}= \begin{cases}\frac{\gamma N-(\gamma-1) \min (N, R)}{\min (N, R)+\beta \max (0, N-R)}, & \text { if } N_{0} \geq N \\ \frac{\left\lfloor N_{0}\right\rfloor \delta-R(\gamma-1)+\beta N}{R+\beta(N-R)}, & \text { if } N_{0}<N\end{cases}
$$

Proof. The social cost under action profile $a$ equals:

$$
\begin{array}{r}
C\left(N_{p u b}(a)\right)=\sum_{i=1}^{N} c_{i}^{N}(a)= \\
c_{p u b, s}\left(N \beta-N_{p u b}(a)(\beta-1)\right), \text { if } N_{p u b}(a) \leq R \text { and } \\
c_{p u b, s}\left(N_{p u b}(a) \delta-R(\gamma-1)+\beta N\right), \text { if } R<N_{\text {pub }}(a) \leq N
\end{array}
$$

The numerators of the two ratios are obtained directly by replacing the first two $N_{p u b}^{N E}$ values (a) and (b) (worst-cases) computed in Theorem 1. On the other hand, under the socially optimal action profile $a_{o p t}$, exactly $R$ drivers pursue on-street parking, whereas the remaining $N-R$ go directly for the private parking. Therefore, under $a_{o p t}$, no drivers find themselves in the unfortunate situation to have to pay the additional cost of cruising in terms of time and fuel after having unsuccessfully competed for an on-street parking spot. The optimal social cost, $C_{o p t}$ is given by:

$$
C_{\text {opt }}=\sum_{i=1}^{N} c_{i}^{N}\left(a_{\text {opt }}\right)=c_{p u b, s}[\min (N, R)+\beta \cdot \max (0, N-R)]
$$

Proposition 2. In the parking spot selection game, the pure Price of Anarchy is upperbounded by $\frac{1}{1-R / N}$ with $N>R$.

Proof. The condition is obtained directly from Proposition 1 when $N>R$. 


\subsection{Mixed-Action Equilibrium Strategies}

We mainly draw our attention on symmetric mixed-action equilibria since these can be more helpful in dictating practical strategies in real systems. Asymmetric mixed-action equilibria are discussed in [14].

Existence: Ashlagi, Monderer, and Tennenholtz proved in ([6], Theorem 1) that a unique symmetric mixed equilibrium exists for the broader family of resource selection games with more than two players and increasing cost functions. It is trivial to repeat their proof and confirm this result for our parking spot selection game $\Gamma$, with $N>R$ and cost functions $w_{p u b}(\cdot)$ and $w_{\text {priv }}(\cdot)$ that are non-decreasing functions of the number of players (increasing and constant, respectively).

Derivation: Let $p=\left(p_{\text {pub }}, p_{\text {priv }}\right)$ denote a mixed-action. Then, the expected costs of choosing the on-street (resp. private) parking space option, when all other drivers play the mixed-action $p$, are given by

$$
\begin{aligned}
c_{i}^{N}(\text { public }, p) & =\sum_{N_{\text {pub }}=0}^{N-1} w_{\text {pub }}\left(N_{\text {pub }}+1\right) B\left(N_{\text {pub }} ; N-1, p_{\text {pub }}\right) \\
c_{i}^{N}(\text { private }, p) & =c_{\text {priv }}
\end{aligned}
$$

where $B\left(N_{p u b} ; N-1, p_{p u b}\right)$ is the Binomial probability distribution with parameters $N-1$ and $p_{p u b}$, for $N_{p u b}$ drivers choosing on-street parking. The cost of the symmetric profile where everyone plays the mixed-action $p$ is given by

$$
c_{i}^{N}(p, p)=p_{\text {pub }} \cdot c_{i}^{N}(\text { public }, p)+p_{\text {priv }} \cdot c_{i}^{N}(\text { private }, p)
$$

With these at hand, we can now postulate the following Theorem.

Theorem 2. The parking spot selection game $\Gamma$ has a unique symmetric mixed-action Nash equilibrium $p^{N E}=\left(p_{\text {pub }}^{N E}, p_{\text {priv }}^{N E}\right)$, where:

$$
\begin{aligned}
& -p_{p u b}^{N E}=1, \text { if } N \leq N_{0} \text { and } \\
& -p_{\text {pub }}^{N E}=\frac{N_{0}}{N}, \text { if } N>N_{0},
\end{aligned}
$$

$$
\text { where } p_{\text {pub }}^{N E}=1-p_{\text {priv }}^{N E} \text { and } N_{0} \in \mathbb{R} \text {. }
$$

Proof. The symmetric equilibrium for $N \leq N_{0}$ corresponds to the pure NE we derived in Theorem 1 To compute the equilibrium for $N>N_{0}$ we invoke the condition that equilibrium profiles must fulfil

$$
c_{i}^{N}\left(\text { public }, p^{N E}\right)=c_{i}^{N}\left(\text { private }, p^{N E}\right)
$$

namely, the costs of each pure action belonging to the support of the equilibrium mixedaction strategy are equal. Hence, from (12), (13) and (15) the symmetric mixed-action equilibrium $p^{N E}=\left(p_{p u b}^{N E}, p_{\text {priv }}^{N E}\right)$ solves the equation

$$
f(p)=-\beta+\sum_{k=0}^{N-1}\left(\gamma-\min \left(1, \frac{R}{k+1}\right) \cdot(\gamma-1)\right) B(k ; N-1, p)=0
$$

A closed-form expression for the equilibrium $p_{p u b}^{N E}$ is not straightforward. However, it holds that:

$$
\lim _{p \rightarrow 0} f(p)=-\beta+1<0 \text { and } \lim _{p \rightarrow 1} f(p)=\delta\left(1-\frac{N_{0}}{N}\right)>0
$$




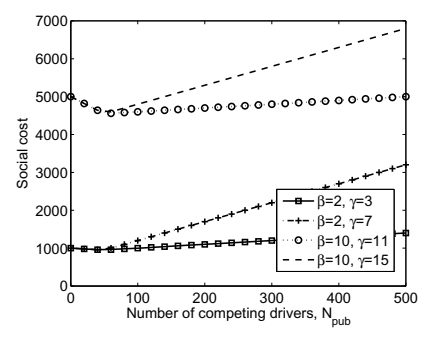

a. Pure-action profiles

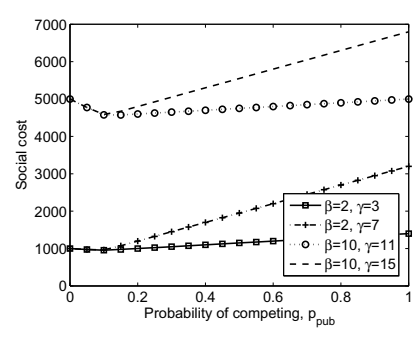

b. Symmetric mixed-action profiles

Fig. 2. Social cost for $N=500$ drivers when exactly $N_{\text {pub }}$ drivers compete (a) or when all drivers decide to compete with probability $p_{p u b}$ (b), for $R=50$ public parking spots, under different charging policies

and $f(p)$ is a continuous and strictly increasing function in $p$ since

$$
f^{\prime}(p)=\sum_{k=0}^{N-1}\left(\gamma-\min \left(1, \frac{R}{k+1}\right)(\gamma-1)\right) B^{\prime}(k ; N-1, p)>\sum_{k=0}^{N-1} B^{\prime}(k ; N-1, p)=0
$$

Hence, $f(p)$ has a single solution. It may be checked with replacement that $f\left(N_{0} / N\right)=0$.

\section{Numerical Results}

The analysis in Section 3 suggests that the charging policy for on-street and private parking space and their relative location, which determines the overhead parameter $\delta$ of failed attempts for on-street parking space, affect to a large extent the (in)efficiency of the game equilibrium profiles. In the following, we illustrate their impact on the game outcome and discuss their implications for real systems.

For the numerical results we adopt per-time unit normalized values used in the typical municipal parking systems in big European cities [2]. The parking fee for public space is set to $c_{p u b, s}=1$ unit whereas the cost of private parking space $\beta$ ranges in $(1,16]$ units and the excess cost $\delta$ in $[1,5]$ units. We consider various parking demand levels assuming that private parking facilities in the area suffice to fulfil all parking requests.

Figure 2 plots the social costs $C\left(N_{p u b}\right)$ under pure (Eq. 11) and $C\left(p_{p u b}\right)$ under mixed-action strategies as a function of the number of competing drivers $N_{p u b}$ and competition probability $p_{p u b}$, respectively, where

$$
\begin{aligned}
C\left(p_{p u b}\right)= & c_{p u b, s} \sum_{n=0}^{N}\left(\begin{array}{c}
N \\
n
\end{array}\right) p_{p u b}^{n}\left(1-p_{p u b}\right)^{N-n} . \\
& {[\min (n, R)+\max (0, n-R) \gamma+(N-n) \beta] }
\end{aligned}
$$

Figure 2 motivates two remarks. First, the social cost curves for pure and mixed-action profiles have the same shape. This comes as no surprise since for given $N$, any value for 


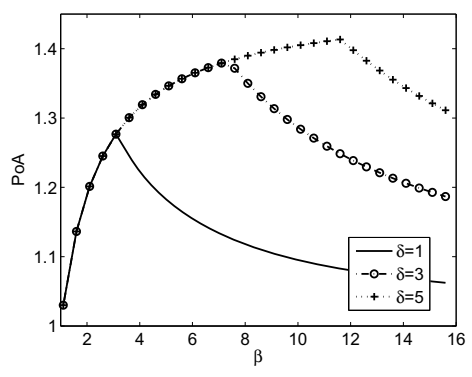

a. $2 D \operatorname{POA}(\beta), R=160$

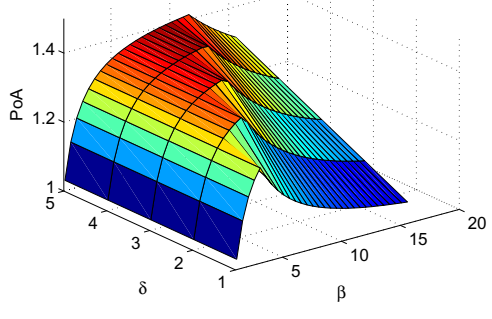

b. $3 D \operatorname{POA}(\beta, \delta), R=160$

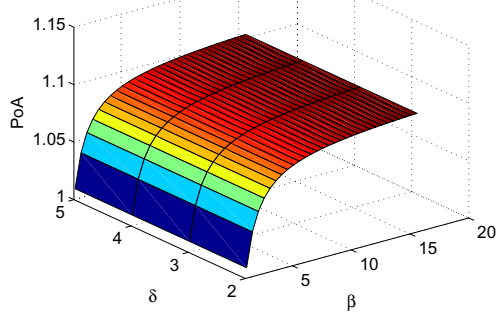

c. $3 D \operatorname{POA}(\beta, \delta), R=50$

Fig. 3. Price of Anarchy for $N=500$ and varying $R$, under different charging policies

the expected number of competing players $0 \leq N_{p u b} \leq N$ can be realized through appropriate choice of the symmetric mixed-action profile $p$. Second, the cost is minimized when the number of competing drivers is equal to the number of on-street parking spots. The cost rises when either competition exceeds the available on-street parking capacity or drivers are overconservative in competing for on-street parking. In both cases, the drivers pay the penalty of the lack of coordination in their decisions. The deviation from optimal grows faster with increasing price differential between the on-street and private parking space.

Whereas an optimal centralized mechanism would assign exactly $\min (N, R)$ public parking spots to $\min (N, R)$ drivers, if $N>R$, in the worst-case equilibrium the size of drivers' population that actually competes for on-street parking spots exceeds the real parking capacity by a factor $N_{0}$ which is a function of $R, \beta$ and $\gamma$ (equivalently, $\delta$ ) (see Lemma 11. This inefficiency is captured in the PoA plots in Figure 3 for $\beta$ and $\delta$ ranging in $[1.1,16]$ and $[1,5]$, respectively. The plots illustrate the following trends:

Fixed $\delta$ - varying $\beta$ : For $N \leq N_{0}$ or, equivalently, for $\beta \geq \frac{\delta(N-R)+R}{R}$, it holds that $\frac{\vartheta P_{O} A}{\vartheta \beta}<0$ and therefore, the POA is strictly decreasing in $\beta$. On the contrary, for $\beta<\frac{\delta(N-R)+R}{R}$, the $\mathrm{POA}$ is strictly increasing in $\beta$, since $\frac{\vartheta P_{o} A}{\vartheta \beta}>0$. 
Fixed $\beta$ - varying $\delta$ : For $N \leq N_{0}$ or, equivalently, for $\delta \leq \frac{R(\beta-1)}{N-R}$ we get $\frac{\vartheta P_{o} A}{\vartheta \delta}>$ 0 . Therefore, the POA is strictly increasing in $\delta$. For $\delta>\frac{R(\beta-1)}{N-R}$, we get $\frac{\vartheta P_{o} A}{\vartheta \delta}=0$. Hence, if $\delta$ exceeds $\frac{R(\beta-1)}{N-R}$, POA is insensitive to changes of the excess cost $\delta$.

Practically, the equilibrium strategy emerging from this kind of assisted parking search behavior, approximates the optimal coordinated mechanism when the operation of private parking facilities accounts for drivers' preferences as well as estimates of the typical parking demand and supply. More specifically, if, as part of the pricing policy, the cost of private parking is less than $\frac{\delta(N-R)+R}{R}$ times the cost of on-street parking, then the social cost in the equilibrium profile approximates the optimal social cost as the price differential between public and private parking decreases. This result is inline with the statement in [16], arguing that "price differentials between on-street and off-street parking should be reduced in order to reduce traffic congestion". Note that the POA metric also decreases monotonically for high values of the private parking cost, specifically when the private parking operator desires to gain more than $\frac{\delta(N-R)+R}{R}$ times the cost of on-street parking, towards a bound that depends on the excess cost $\delta$. Nevertheless, these operating points correspond to high absolute social cost, i.e., the minimum achievable social cost is already unfavorable due to the high fee paid by $N-R$ drivers that use the private parking space (see Fig. 2, large $\beta$ ). On the other hand, there are instances, as in case of $R=50$ (see Fig. 3), where the value $\frac{\delta(N-R)+R}{R}$ corresponds to a non-realistic option for the cost of private parking space, already for $\delta>1$. Thus, contrary to the previous case, POA only improves as the cost for private parking decreases. Finally, for given cost of the private parking space, the social cost can be optimized by locating the private facility in the proximity of the on-street parking spots so that the additional travel distance is reduced to the point of bringing the excess cost below $\frac{R(\beta-1)}{N-R}$.

\section{Related Work}

Various aspects of the broader parking space search problem have been addressed in the literature. Probably the first formulation of the problem appeared in [17] in the context of the broader family of stopping problems. Parking spots are spread randomly, with density $\lambda$ over equal-size blocks that are adjacent to a destination, and drivers circle through them crossing the destination every time such a circle is over. Ferguson in [11] considers a simpler variant of the problem, whereby the drivers' destination lies in the middle of an infinite-length straight line with parking spots that are occupied with probability $p$. In either case, the optimal policy for the drivers is shown to be of the threshold type: they should occupy an available vacant parking spot whenever this lies within some distance $r=f(\lambda)$, resp. $f(p)$, from their destination and continue searching otherwise.

Pricing and more general economic dimensions of the parking allocation problem are analyzed in [16] and [3]. A queueing model for drivers who circulate in search for onstreet parking is introduced in [16] in order to analyze economic effects of congestion pricing. From a microeconomical point of view, Anderson and de Palma in [3] treat the parking spots as common property resource and question whether free access or some 
pricing structure result in more efficient use of the parking capacity. Working on a simple model of city and parking spot distribution, they show that this use is more efficient (in fact, optimal) when the spots are charged with the fee chosen in the monopolistically competitive equilibrium with private ownership. The situation is reversed, i.e., drivers are better off when access to the parking spots is free of charge.

Subsequent research contributions in [5], [4] and [7] have explicitly catered for strategic behavior and the related game-theoretic dimensions of general parking applications. In [5], the games are played among parking facility providers and concern the location and capacity of their parking facility as well as which pricing structure to adopt. Whereas, in the other two works, the strategic players are the drivers. In [4], which seeks to provide cues for optimal parking lot size dimensioning, the drivers decide on the arriving time at the lot, accounting for their preferred time as well as their desire to secure a space. In a work more relevant to ours, the authors in [7] define a game setting where drivers exploit (or not) information on the location of others to occupy an available parking spot at the minimum possible travelled distance, irrespective of the distance between the spot and driver's actual travel destination. The authors present distributed parking spot assignment algorithms to realize or approximate the Nash equilibrium states. In our work, the game-theoretic analysis highlights the cost effects of the parking operators' pricing policies on drivers' decisions, drawing on closed-form expressions of the stable operational points in the different game settings.

\section{Conclusions - Discussion}

In this paper, we draw our attention on fundamental determinants of parking assistance systems' efficiency rather than particular realizations. We have, thus, formulated the information-assisted parking search process as an instance of resource selection games to assess the ultimate impact that an ideal mechanism broadcasting accurate information on parking demand, supply and pricing policies, can have on drivers' decisions. Our model dictates plausible conditions under which different pricing policies steer the equilibrium strategies, reduce the inefficiency of the parking search process, and favor the social welfare.

We conclude by iterating on the strong and long-debatable assumption that drivers do behave as fully rational utility-maximizing decision-makers; namely, they can exhaustively analyze the possible strategies available to themselves and the other drivers, identify the equilibrium profile(s), and act accordingly to realize it. Simon in [19], challenged both the normative and descriptive capacity of the fully rational decision-maker, arguing that human decisions are most often made under knowledge, time and computational constraints. One way to accommodate the first constraints is through (pre-) Bayesian games of incomplete information. In [14], we formulate (pre-)Bayesian variants of the parking spot selection game to assess the impact of information accuracy on drivers' behavior and, ultimately, the service cost. However, models that depart from the utility-maximization norm and draw on fairly simple cognitive heuristics, e.g., [12], reflect better Simon's argument that humans are satisficers rather than maximizers. For example, the authors in [13] explore the impact of the fixed-distance heuristic on a simpler version of the unassisted parking search problem. The comparison of normative and more descriptive decision-making modeling approaches both in the context of 
the parking spot selection problem and more general decision-making contexts, is an interesting area worth of further exploration.

\section{References}

[1] http://sfpark.org/

[2] http://www.city-parking-in-europe.eu/

[3] Anderson, S.P., de Palma, A.: The economics of pricing parking. Journal of Urban Economics 55(1), 1-20 (2004)

[4] Arbatskaya, M., Mukhopadhaya, K., Rasmusen, E.: The parking lot problem. Tech. rep., Department of Economics, Emory University, Atlanta (2007)

[5] Arnott, R.: Spatial competition between parking garages and downtown parking policy. Transport Policy (Elsevier), 458-469 (2006)

[6] Ashlagi, I., Monderer, D., Tennenholtz, M.: Resource selection games with unknown number of players. In: Proc. AAMAS 2006, Hakodate, Japan (2006)

[7] Ayala, D., Wolfson, O., Xu, B., Dasgupta, B., Lin, J.: Parking slot assignment games. In: Proc. 19th ACM SIGSPATIAL GIS (2011)

[8] Caliskan, M., Graupner, D., Mauve, M.: Decentralized discovery of free parking places. In: Proc. 3rd VANET (in Conjunction with ACM MobiCom), Los Angeles, CA, USA (2006)

[9] Cheng, S.G., Reeves, D.M., Vorobeychik, Y., Wellman, M.P.: Notes on the equilibria in symmetric games. In: Proc. 6th Workshop on Game Theoretic and Decision Theoretic Agents (Colocated with IEEE AAMAS), New York, USA (August 2004)

[10] Delot, T., Cenerario, N., Ilarri, S., Lecomte, S.: A cooperative reservation protocol for parking spaces in vehicular ad hoc networks. In: Proc. 6th Inter. Conference on Mobile Technology, Application and Systems, MOBILITY (2009)

[11] Ferguson, T.S.: Optimal stopping and applications, http://www.math.ucla.edu/ tom/Stopping/

[12] Goldstein, D.G., Gigerenzer, G.: Models of ecological rationality: The recognition heuristic. Psychological Review 109(1), 75-90 (2002)

[13] Hutchinson, J.M.C., Fanselow, C., Todd, P.M.: Ecological rationality: intelligence in the world. Oxford University Press, New York (2012)

[14] Kokolaki, E., Karaliopoulos, M., Stavrakakis, I.: Leveraging information in vehicular parking games. Tech. rep., Dept. of Informatics and Telecommunications, Univ. of Athens (2012), http://cgi.di.uoa.gr/ evako/tr1.pdf

[15] Koutsoupias, E., Papadimitriou, C.H.: Worst-case equilibria. Computer Science Review 3(2), 65-69 (2009)

[16] Larson, R.C., Sasanuma, K.: Congestion pricing: A parking queue model. Journal of Industrial and Systems Engineering 4(1), 1-17 (2010)

[17] MacQueen, J., Miller, J.: Optimal persistence policies. Operations Research 8(3), 362-380 (1960)

[18] Mathur, S., Jin, T., Kasturirangan, N., Chandrasekaran, J., Xue, W., Gruteser, M., Trappe, W.: Parknet: Drive-by sensing of road-side parking statistics. In: Proc. 8th ACM MobiSys., San Francisco, CA, USA, pp. 123-136 (2010)

[19] Simon, H.A.: A behavioral model of rational choice. The Quarterly Journal of Economics 69(1), 99-118 (1955)

[20] Wang, H., He, W.: A reservation-based smart parking system. In: Proc. 5th International Workshop on Cyber-Physical Networking Systems (Colocated with IEEE INFOCOM) (April 2011) 